\title{
Database analysis and visualization of simulated and recorded electrophysiological data with PANDORA's Toolbox in Matlab Cengiz Günay* and Dieter Jaeger
}

\author{
Address: Biology Department, Emory University, Atlanta \\ Email: Cengiz Günay* - cgunay@emory.edu \\ * Corresponding author
}

from Seventeenth Annual Computational Neuroscience Meeting: CNS*2008

Portland, OR, USA. 19-24 July 2008

Published: II July 2008

BMC Neuroscience 2008, 9(SuppI I):P82 doi:I0.1 I86/I47I-2202-9-SI-P82

This abstract is available from: http://www.biomedcentral.com/I47I-2202/9/SI/P82

(C) 2008 Günay and Jaeger; licensee BioMed Central Ltd.

\section{Introduction}

We developed a generic database-supported analysis and visualization software, PANDORA, for research projects with large datasets and many parameters. PANDORA supports analyzing electrophysiological data from intracellular recordings and from computer simulations of compartmental neuron models. An easy querying system matches the power of SQL databases, while the Matlab computing environment provides specialized numeric processing, analysis and visualization tools. PANDORA can be used for organizing and keeping track of complex physiological (e.g., recording channel, stimulus magnitude) or computer simulation (e.g., ion channel densities, kinetics, compartment lengths) parameters of a single neuron or network model. The PANDORA toolbox can be freely obtained from http://userwww.service.emory.edu/ $\simeq$ cgunay/pandora.

\section{Motivation}

The amount of electrophysiological data is increasing as more channels can be sampled and recording quality improves, while rapid advances in computing speed and capacity have enabled researchers to generate massive amounts of simulation data in very short times. Recently, interesting results were obtained from modeling studies with such large datasets $[1,2]$.

Although widely used for identified neurons and brain connectivity [3], databases are rarely used in electrophysiological analysis [4]. The main advantage of using a database is being able to associate metadata labels with raw data for querying and organizing the data based on the information in these labels. For example, automatic labeling of control and drug applied recordings in a database reduces risk of analysis errors.

\section{Results}

PANDORA offered specific improvements in analyzing electrophysiological data. Neuroscientists conventionally prefer qualitative analysis of raw data traces. However, as the amount of collected data increases, it is more desirable to have quantitative results. PANDORA offers an automated way of extracting measurements defined by the electrophysiologist. It was more efficient to storage, search and analyze these measurements and to find associated experimental or simulation parameters. Furthermore, PANDORA kept pointers back to raw data that allowed verifying results obtained from this high-level analysis.

Electrophysiological data sometimes needs to be analyzed at different levels of abstraction. At the lower level, multiple traces collected from one neuron must be displayed and analyzed, while to understand effects across neurons, one must look at summary information from each neuron. PANDORA routines that allow one to sift, average and collapse parameter dimensions were essential in switching between these levels of abstraction.

For large simulation projects searching model parameters, PANDORA offered several routines to understand the effects, on the measured characteristics, of a single parameter while other parameters were invariant. The results could then be subjected to second tier analyses such as derivative and correlation, or simply be plotted. 
In summary, PANDORA was aimed to make analysis of electrophysiological data easier and provides a flexible platform for standardized analysis.

\section{Acknowledgements}

This work was supported by NINDS ROI-NS039852 and NIMH ROIMH065634.

\section{References}

I. Prinz AA, Bucher D, Marder E: Similar network activity from disparate circuit parameters. Nature Neurosci 2004, 7(12): I345-I352.

2. Günay $C$, Edgerton JR, Jaeger $D$ : Channel density distributions explain spiking variability in the globus pallidus: $A$ combined physiology and computer simulation database approach. 2008. In review

3. Pittendrigh S, Jacobs G: Neurosys - a semistructured laboratory database. Neuroinformatics 2003, I:167-176.

4. Lytton WW: Neural query system: data-mining from within the Neuron simulator. Neuroinformatics 2006, 4(2): 163-175.

Publish with Bio Med Central and every scientist can read your work free of charge

"BioMed Central will be the most significant development for disseminating the results of biomedical research in our lifetime. "

Sir Paul Nurse, Cancer Research UK

Your research papers will be:

- available free of charge to the entire biomedical community

- peer reviewed and published immediately upon acceptance

- cited in PubMed and archived on PubMed Central

- yours - you keep the copyright

Submit your manuscript here:

http://www.biomedcentral.com/info/publishing_adv.asp 